\title{
Polidactilia y asimetría fluctuante en el "loro hablador" (Amazona aestiva): ¿signos de estrés del desarrollo?
}

\author{
Herrera, J.M.'; Berkunsky, I. ${ }^{2}$ \\ ${ }^{1}$ Facultad de Ciencias Veterinarias, ${ }^{2}$ Instituto Multidisciplinario sobre Ecosistemas y Desarrollo Sustentable. \\ Univ.Nac.del Centro Pcia.Buenos Aires, Paraje Arroyo Seco s/n, B7000GHG, Tandil, Argentina. \\ E-mail: jumherrera@gmail.com
}

\begin{abstract}
Resumen
Herrera, J.M.; Berkunsky, I.: Polidactilia y asimetría fluctuante en el "loro hablador" (Amazona aestiva): ¿signos de estrés del desarrollo? Rev. vet. 28: 1, 62-64, 2017. Algunas anormalidades, como la polidactilia y asimetría fluctuante, ocurren como resultado de estrés durante el desarrollo. Se reporta un caso de signos de estrés del desarrollo que incluye polidactilia, asimetría fluctuante y barras de estrés en un pichón de "loro hablador" (Amazona aestiva). Se considera que estas anormalidades estarían relacionadas al estrés del pichón durante un evento de incendio de pastizales. Se examinaron dos pichones silvestres encontrados en un nido afectado por un incendio. El examen clínico mostró anormalidades sólo en el pichón de menor tamaño. Tales anomalías incluyeron a) polidactilia en el miembro posterior derecho; b) marcada asimetría en la coloración de las alas, con una patrón atípico en el ala derecha; y c) barras de estrés en el área de pigmentación roja de plumas secundarias en ambas alas. Los incendios forestales deberían ser considerados un factor de estrés importante para aves que nidifican en cavidades en las Sabanas del Beni (norte de Bolivia).
\end{abstract}

Palabras clave: loro, polidactilia, asimetría fluctuante, estrés del desarrollo, incendio.

\begin{abstract}
Herrera, J.M.; Berkunsky, I.: Polydactyly and fluctuating asymmetry in a wild bluefronted Amazon nestling (Amazona aestiva): clinical signs of developmental stress? Rev. vet. 28: 1, 62-64, 2017. Some abnormalities such as polydactyly and fluctuating asymmetry occur as a result of developmental stress. A case of developmental stress that includes polydactyly, fluctuating asymmetry, and fault bars in one Blue-fronted Amazon nestling ( $\mathrm{Ama}$ zona aestiva), is reported. These anomalies were related to the stress caused by a wildland fire. Two nestlings of a same nest were examined. Physical examination showed anomalies only in the small nestling which included a) polydactyly in the right leg, b) colour asymmetry in wing feathers with an atypical staining pattern in the right wing and c) stress bars in red pigmentation area of secondary feathers of both wings. Wildland fires should be considered as a major stress factor for nesting birds of the Beni Savanna (north of Bolivia).
\end{abstract}

Key words: parrot, polydactyly, fluctuant asymmetry, developmental stress, fire.

\section{INTRODUCCIÓN}

En los animales existen anomalías manifestadas como consecuencia de estrés durante el desarrollo. Esto se debe a la tendencia del sistema para producir cambios morfológicos en respuesta a perturbaciones aleatorias, lo cual se denomina inestabilidad del desarrollo o ruido del desarrollo ${ }^{5,10}$. Tales desórdenes pueden ser provocados por agentes estresantes, por deficiencia alimentaria o por contaminantes ${ }^{3,6,22}$.

La presentación de polidactilia en aves se ha reportado con diversas etiologías (factores físicos, químicos o genéticos), siempre afectando al individuo en el pe-

Recibido: 16 agosto 2016 / Aceptado: 28 noviembre 2016 riodo embrionario ${ }^{4,18}$. Se afirma que por acción de un estrés muy intenso, la homeostasis puede alterarse durante el desarrollo y permitir la expresión de mutaciones ${ }^{5}$.

En el caso de los rasgos bilaterales simétricos, las desviaciones pequeñas y aleatorias se denominan asimetría fluctuante ${ }^{21}$. Tal anomalía aumentaría con el estrés ambiental y/o genético ${ }^{13}$ y podría estar relacionada con el fitness y la calidad de los individuos ${ }^{3,12}$.

En las aves, perturbaciones como enfermedades, malnutrición y estrés ambiental, pueden manifestarse en las plumas, ya que durante su formación una hipoproteinemia lleva a la interrupción en el depósito de aminoácidos ${ }^{9}$. Esto genera una despigmentación conocida como barras de estrés, la cual se manifiesta vi- 
sualmente como una "ruptura" transversal en el vexilo de la pluma. La presencia de muchas plumas con barras de estrés es indicativa de un problema en el pasado reciente del ave ${ }^{20}$.

En el presente artículo se reporta un caso de presentación de signos de perturbación durante el desarrollo en un pichón de "loro hablador" (Amazona aestiva). Se propone que las anomalías observadas estarían vinculadas al estrés sufrido por el individuo durante su desarrollo, consecuencia de un incendio forestal que afectó al nido durante el período de incubación de los huevos.

\section{MATERIAL Y MÉTODOS}

Como parte de un proyecto de conservación en marcha, se monitorearon nidos de loros salvajes en las Sabanas del Beni (también llamadas Llanos de Moxos), en el norte de Bolivia. Ésta es un área de $160.000 \mathrm{~km}^{2}$ temporalmente inundada, cubierta por una sabana sin árboles, interpuesta con un complejo mosaico de islas y galerías ribereñas de bosques, donde la principal actividad económica es la cría de ganado ${ }^{11}$.

Durante septiembre de 2010, un gran incendio forestal afectó más de $400 \mathrm{~km}^{2}$ del área de estudio. Se visitó esta zona un mes más tarde, encontrándose un nido de "loro hablador" en una cavidad localizada a 5,8 $\mathrm{m}$ de altura sobre una palmera "mbocayá" (Acrocomia aculeata; $13^{\circ} 49^{\circ} \mathrm{S}, 64^{\circ} 31^{\prime} \mathrm{W}$ ). El árbol se hallaba en el límite entre una isla y el pastizal, y mostraba claros signos de impacto del incendio (hojas y tronco quemados).

Dentro del nido, se encontraron dos pichones de aproximadamente 45 días. Ambos individuos fueron examinados a campo y se les tomaron fotografías para ser luego analizadas en laboratorio.

\section{RESULTADOS}

Al examen físico se registraron anomalías en una pata y en plumas secundarias del pichón de menor tamaño, mientras que el mayor permaneció normal. Las anormalidades incluyeron: a) polidactilia en el miembro pelviano derecho; b) marcada asimetría de color en plumas de las alas, con un patrón de coloración atípico en el miembro torácico derecho; y c) barras de estrés en plumas secundarias de ambos miembros torácicos.

El miembro pelviano derecho presentó polidactilia. El quinto dedo se ubicó hacia lateral del dígito 1, con una longitud menor (Figura 1).

Se observó asimetría en la pigmentación de plumas secundarias. En el ala izquierda, las plumas mostraron la configuración típica, con las barbas externas color amarillo en la base de la pluma, luego rojo, verde y por último azul, y las barbas internas con coloración uniforme negra (Figura 2, izquierda).

En cambio, las plumas del ala derecha mostraron una alteración en el patrón de coloración tanto para las barbas externas como internas. Las barbas externas mostraron rojo en la base de la pluma (en lugar de amarillo), luego amarillo (en lugar de verde), verde y por úl-

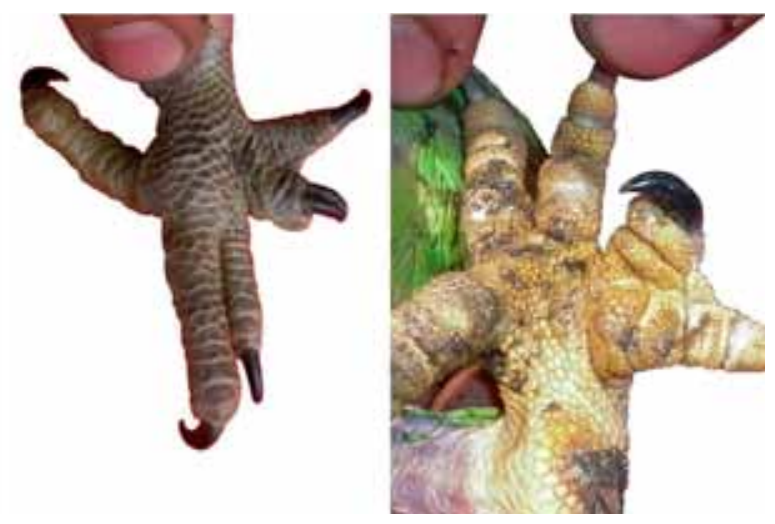

Figura 1. Vista dorsal y plantar del miembro pelviano derecho del pichón de "loro hablador" (Amazona aestiva) con polidactilia.

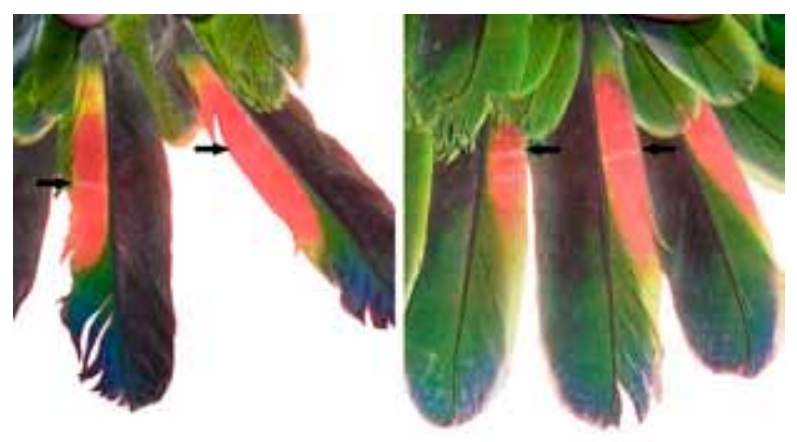

Figura 2. Las plumas del ala izquierda muestran el patrón de coloración típico, mientras que las del ala derecha exhiben un patrón alterado. Las flechas indican "barras de estrés".

timo azul. En las barbas internas se observó coloración verde y azul (en lugar de negra) hacia el extremo distal de la pluma (Figura 2, derecha).

Las barras de estrés se observaron en plumas secundarias de ambas alas, observándose como bandas de despigmentación en dirección perpendicular al raquis de la pluma, en la barba externa y exclusivamente en el área de pigmentación roja de la pluma (Figura 2).

\section{DISCUSIÓN}

El número y posición de los dígitos o falanges de las aves es un carácter hereditario estable ${ }^{18}$. Desviaciones en su número y posición han sido reportadas en muchas especies de aves, en su mayoría de forma simétrica, tanto en miembros torácicos como pelvianos. Sin embargo, sólo existe un reporte de polidactilia en un ave psitácida, la cual, al igual que en el presente caso, era de presentación unilateral ${ }^{4}$.

Así como se ha reportado la posible etiología genética, también se postula que factores externos, tanto físicos como químicos, podrían estar involucrados en esta malformación ${ }^{18}$. Entre éstos, la temperatura ambiental elevada durante el desarrollo embrionario es considerada como un posible factor inductor de polidactilia, junto a otras malformaciones. Los pichones de "loro hablador" estudiados todavía estaban incubando 
durante los incendios, por lo que asumimos que fueron expuestos a altos niveles de estrés durante el desarrollo embrionario. Así, la polidactilia observada en el pichón se podría explicar como resultado de la exposición a las altas temperaturas.

En años recientes, dos bioindicadores han sido utilizados para evaluar la calidad de condiciones en el ambiente en estudios de aves: la asimetría bilateral o fluctuante ${ }^{2}$ y las barras de estrés ${ }^{1}$. La asimetría fluctuante es utilizada para comparar los niveles de inestabilidad del desarrollo en poblaciones sometidas a factores de estrés ambiental ${ }^{8,16}$. Según las hipótesis propuestas, se observarían mayores niveles de asimetría fluctuante en poblaciones muy expuestas a factores de estrés, que en aquellas poco expuestas ${ }^{7,14}$.

Las barras de estrés en aves son anormalidades de crecimiento de las plumas, comunes y bien conocidas, que debilitan la pluma, incrementando la posibilidad del quiebre ${ }^{19} \mathrm{y}$ afectando seriamente el fitness de las aves al perjudicar su capacidad de vuelo ${ }^{1,15}$. Tanto la asimetría fluctuante como las barras de estrés son señales de un desarrollo anormal. Sin embargo, mientras la asimetría fluctuante se relaciona con estrés a largo plazo, lo cual perturba el desarrollo e incita a la inestabilidad del mismo, las barras de estrés son más probablemente un resultado de agentes de estrés agudos ${ }^{17}$.

Se ha reportado que la polidactilia del ave podría ser causante de alteraciones de la postura y la locomoción, lo cual le generaría estrés ${ }^{4}$. Esta hipótesis podría aplicarse al presente caso en estudio. La polidactilia observada sería la causante del estrés que llevó al individuo a presentar asimetría fluctuante y barras de estrés.

En conclusión, se estima que los incendios de pastizales deberían considerarse como un factor de estrés para las aves que nidifican en las islas, especialmente en los árboles periféricos. Nuevos estudios son necesarios para profundizar los conocimientos en relación a los incendios como factor de perturbación y su asociación con el estrés en pichones de aves. Estos estudios podrían contribuir al diseño de tácticas de manejo de incendios de pastizal para evitar los efectos sobre las islas de bosques, como una acción para optimizar la conservación del "loro hablador" en las Sabanas del Beni.

\section{REFERENCIAS}

1. Bortolotti GR, Dawson RD, Murza GL. 2002. Stress during feather development predicts fitness potential. $J$ Anim Ecol 71: 333-342.

2. Brown CR, Brown MB. 1998. Intense natural selection on body size and wing and tail asymmetry in "cliff swallows" during severe weather. Evolution 52: 1461-1475.

3. Clarke GM. 1995. Relationships between developmental stabiality and fitness: application for conservation biology. Conserv Biol 9: 18-24.

4. Crosta L, Bürkle M, Timossi L, Kaleta EF. 2002. Unilateral pentadactyly in a yellow-shouldered amazon ( $\mathrm{Ama}$ zona barbadensis). J Avian Med Surg 16: 26-30.
5. Dongen S. 2006. Fluctuating asymmetry and developmental instability in evolutionary biology: Past, present and future. J Evol Biol 19: 1727-1743.

6. Graham JH, Emlen JM, Freeman DC. 1993. Developmental stability and its applications in ecotoxicology. Ecotoxicology 2: 175-184.

7. Graham JH, Fletcher D, Tigue J, McDonald M. 2000. Growth and developmental stability of Drosophila melanogaster in low frequency magnetic fields. Bioelectromagnetics 21: 465-472.

8. Hoffmann AA, Woods RE. 2003. Associating environmental stress with developmental stability: problems and patterns. In: Developmental instability: causes and consequences (Polak M ed.), Oxford Univ. Press, Oxford, p. 387-401.

9. Jiménez J. 2008. Avian dermatology: more than feather picking. Proceed Southern Europ Vet Conf \& Congr Nac AVEPA, Barcelona, España.

10. Klingenberg CP. 2003. Developmental perspective on developmental instability: theory, models and mechanisms. In: Developmental instability: causes and consequences (Polak M ed.), Oxford Univ. Press, Oxford, p. 14-34.

11. Mayle FE, Langstroth RP, Fisher RA, Meir P. 2007. Long-term forest-savannah dynamics in the bolivian amazon: implications for conservation. Philos Trans $R$ Soc Lond B Biol Sci 362: 291-307.

12. Møller AP, Hoglund J. 1991. Patterns of fluctuating asymmetry in avian feather ornaments: Implications for models of sexual selection. Proc R Soc B Biol Sci 245: 1-5.

13. Palmer AR, Strobecke C. 1986. Fluctuating asymmetry: measurement, analysis, patterns. Ann Rev Ecol Syst 17: 391-421.

14. Pankakoski E, Koivisto I, Hyvarinen H. 1992. Reduced developmental stability as an indicator of heavy metal pollution in common shrew Sorex avensis. Acta Zool Fenn 191: 137-144.

15. Pap PL, Barta Z, Tökö lyi J, Vágási IC. 2007. Increase of feather quality during moult: A possible implication of feather deformities in the evolution of partial moult in the great tit Parus major. J Avian Biol 38: 471-478.

16. Parsons PA. 1992. Fluctuating asymmetry: a biological monitor of environmental and genomic stress. Heredity 68: 361-364.

17. Prentice S, Jobes AP, Burness G. 2008. Fault bars and fluctuating asymmetry in birds: Are the two measures correlated? J F Ornithol 79: 58-63.

18. Rieck GW, Herzog A. 1984. General veterinary genetics, cytogenetics and general teratology, Ferdinand Enke Verlag, Stuttgar, $352 \mathrm{p}$.

19. Sarasola JH, Jovani R. 2006. Risk of feather damage explains fault bar occurrence in a migrant hawk, the Swainson's hawk Buteo swainsoni. J Avian Biol 37: 29-35.

20. Schmidt RE, Lightfoot TL. 2005. Integument. In: Clinical Avian Medicine (Harrison G, Lightfoot T, eds.), Spix Publishing, Palm Beach, p. 395-410.

21. van Valen L. 1962. A study of fluctuating asymmetry. Int J Org Evol 53: 160.

22. Watson PJ, Thornhill R. 1994. Fluctuating asymmetry and sexual selection. Trends Ecol Evol 9: 21-25. 